\title{
Diferencias y convergencias entre el pragmatismo peirceano y otros pragmatismos
}

Paniel Reyes

Universidad Popular Autónoma del Estado de Puebla

Resumen: Distintas doctrinas, incluso contrapuestas entre sí, presumen de ser una forma de "pragmatismo". Para el fundador del Pragmatismo en tanto tradición filosófica, Charles Peirce, esta situación puede resultar en una lamentable confusión y, por tanto, acuñó el término de 'pragmaticismo' para distinguir su particular versión. Sin embargo, Peirce mismo no escribió un texto sistemático en el que explicara qué criterios específicos distinguen su pragmatismo de cualquier otro. En este texto presento algunos de estos criterios que me parecen fundamentales: (I) el uso de una máxima pragmática sobre la cual ha de descansar el principio lógico que fundamenta la tradición, (2) La necesidad de adoptar el realismo escolástico y rechazar el nominalismo; y (3) El caracter operacionalista, opuesto al inferencialista, del pragmatismo de Peirce. Además, presento algunas opiniones que hacen pensar que a pesar de las distinciones que estos criterios establecen hay un núcleo de convergencia entre los distintos tipos de pragmatismos presentes en la tradición filosófica.

Palabras clave: pragmatismo, operacionalismo, máxima pragmática, Charles Peirce, William James.

Abstract: The term "pragmatism" has been used and abused by doctrines that are even contradictory among them. For the founder of Pragmatism as a philosophical tradition, Charles Peirce, this situation might seem a sad misunderstanding, and that is why he came up with the term "pragmaticism" in order to distinguish his own particular version of Pragmatism. However, Peirce himself did not leave a systematic treaty that explained what kind of specific conditions distinguish his pragmatism from other versions. In this essay I introduce some criteria that I deem fundamental to disambiguate the meaning of Pragmatism: (I) the use of the logical principle known as the 'pragmatic maxim', upon which the corner stone of the tradition should rest; 
(2) the need to adopt Scholastic Realism and reject Nominalism; and (3), the operationalist character (as opposed to the inferential character) of Peirce's pragmatism. In addition, I offer some views avowing that in spite of the differences and distinctions that these criteria establish, there is a core of convergence between the different kinds of pragmatisms present in the philosophical tradition.

Keywords: pragmatism, operationalism, pragmatic maxim, Charles Peirce, WilliamJames.

\section{$\S 1$. Pragmatismos}

El término pragmatismo ha sido usadoy abusado continuamente y ciertamente se encuentra en una racha de bonanza en los círculos filosóficos: se habla, como lo establece Richard Bernstein, de un "giro pragmatista” de la filosofía. Sin embargo, ya en tiempos de los tres pragmatistas clásicos Peirce, James y Dewey hubo un debate sobre el significado del término y su interpretación correcta. El debate contemporáneo acerca del pragmatismo, como bien ha señalado Susan Haack, también tiene dos interpretaciones que se excluyen entre sí: Haack llama pragmatismo en sentido propio a la tradición iniciada e inspirada por Peirce, mientras que expresa sus preocupaciones por usos tan libres del término en las filosofías de Rorty y posiblemente Brandom, llamándoles "pragmatismo vulgar”.

Sabemos que Peirce quería desligarse de la ambigüedad generada en la interpretación de su pragmatismo presente en algunos comentadoresy popularizada como una actitud y ethos. Sin embargo, Peirce no negó rotundamente que existiera un núcleo pragmatista en otras versiones del pragmatismo, más bien, se lamentaba de la ausencia del interés lógico y sobre todo de las tendencias nominalistas de las otras versiones. En efecto, para Peirce el pragmatismo solo puede ser abrazado si se aceptan los aspectos recalcitrantes de una realidad independiente de nuestra idiosincrasia y, por tanto, requiere la adopción de su realismo escolástico extremo.

El propósito, pues, de estas líneas es poner de manifiesto un conjunto de ideas sobre el pragmatismo en sentido amplio y estricto. Mi tesis es que el pragmatismo amplio beneficia a la filosofía cuando se hace conciencia de su 
relación con el pragmatismo estricto. El pragmatismo estricto, por su parte, es el resultado de la adopción de una máxima que clarifica nuestras ideas y difícilmente se puede desambiguar sin el uso de tal principio lógico.

\section{§ 2. Pragmatismo amplio}

Richard Bernstein' presentó de manera particularmente clara los cinco temas que suelen identificar el pragmatismo en sentido amplio:

I. Anti-fundacionalismo.

2. Falibilismo militante.

3. Énfasis en el carácter social del "yo" y la necesidad de alimentar una comunidad de investigación.

4. Reconocimiento de la ubiquidad de la contingencia y el azar y, por tanto, reconocimiento de la precariedad de la experiencia.

5. Pluralismo con respecto a las tradiciones, perspectivas y orientaciones en la filosofía, la ciencia y la cultura ampliamente concebidas.

Bernstein identifica estos elementos en los pragmatistas clásicos como temas comunes en Peirce, James, Dewey y Mead. Un problema que emerge de las consideraciones de Bernstein, sin embargo, radica en la pregunta consiguiente a si estos criterios son condiciones necesarias y suficientes para ser un pragmatista, o si estas condiciones tienen que cumplirse en cada caso y si otros temas pueden caracterizar la actitud pragmatista, o si alguna de las características sobresale de todas las demás: es necesario, pues desambiguar el pragmatismo para que finalmente pueda ser una contribución a la discusión filosófica con una identidad específica. Autores como Burke ${ }^{2}$ o Talisse ${ }^{3}$ tienden a coincidir en la idea de que el pragmatismo se distingue por un proceso.

\footnotetext{
Cf. Bernstein, Richard, The pragmatic turn, Cambridge: Polity Press, 2010.

2 Cf. Burke, Thomas, What Pragmatism Was? Indiana: Indiana University Press, 2013.

3 Cf. Talisse, Robert, Democracy and Moral Conflict, Cambridge: Cambridge University Press, 2010.
} 


\section{$\S 3$. Operacionalismo}

El principio operacionalista es que el significado de un término debe regirse bajo un criterio realista que puede funcionar como su medida o verificación, sea este capturado bajo el concepto de operación o bajo el de evidencia empírica u observable. Bajo esta doctrina, sin embargo, caben varias posturas que reconocen dicho criterio realista con distintos tonos.

El operacionalismo más notable durante el siglo XX fue el del físico Bridgman. Para Bridgman el conocimiento es legítimo si y solo si puede ser derivado en operaciones de los conceptos. Bridman definía el opreacionalismo de este modo: "(...) we mean by any concept nothing more than a set of operations; the concept is synonymous with the corresponding set of operations". El operacionalismo tradicional de los físicos de la primera mitad del siglo $X X$ entró en una crisis profunda cuando la Teoría General de la Relatividad propuso un concepto de operación más allá de las operaciones verificables.

El operacionalismo de Peirce vinculado al pragmatismo, sin embargo, no implica un concepto de operación como verificación de un hecho concreto, sino que acepta cierto grado de vaguedad en el concepto de consecuencia práctica. En efecto, el realismo de Peirce es primariamente un realismo de regularidades que son instanciadas con variaciones infinitesimales. Pensemos en un problema clásico propuesto al pragmatismo: el problema de los hechos perdidos (lost facts or buried secrets). Una manera de considerar este problema desde el punto de vista del realismo de Peirce es que aunque hechos en particular han quedado más allá de nuestro acceso a la evidencia, con todo la investigación puede proseguir señalándonos una dirección hacia la que los hechos pueden dirigirse: es decir, la investigación tal vez ha perdido el acceso a información concreta sobre el clima en la mañana en que Bruto asesinó a César, pero los patrones y hábitos que podemos descubrir al estudiar el clima nos pueden sorprender con acercamientos y probabilidades continuadas. 


\section{$\S 4$. Inferencialismo}

El pragmatismo se ha distinguido no sólo por su referencia a las operaciones, sino también a la importancia de los aspectos contextuales de los conceptos. Así, por ejemplo, el pragmatismo de James identificó la máxima pragmática no solo como un principio lógico, sino como una actitud que ayuda a entender el contexto de nuestros significados a la hora de resolver conflictos de tipo filosófico. En efecto, en 1907 encontramos a James introduciendo la máxima de Peirce en Pragmatism: A New Name for some Old ways of Thinking: en esta obra, James omite algunos elementos de la relación entre duda y creencia de Peirce $y$, más bien, se preocupa por ofrecer el pragmatismo como una "filosofía mediadora" entre temperamentos opuestos.

James desarrolló ideas en torno a la verdad que adquirieron un tono inferencialista, especialmente al desarrollar su teoría pragmática sobre el expediente de la verdad: "Ideas (...) become true just in so far as they help us to get into satisfactory relations with other parts of our experience" 5 . "Any idea upon which we can ride (...); any idea that will carry us prosperously from any one part of our experience to any other part, linking things satisfactorily, working securely, saving labor; is true for just so much, true in so far forth, true instrumentally"6.

Filósofos como Brandom denotan la estrategia inferencialista como la marca particular del pragmatismo. En efecto, para ellos el pragmatismo es una opción filosófica que se sigue de aceptar que la coherencia de ciertos conceptos conlleva el criterio fundamental de su adopción, o como lo expresa Robert Brandom, establecer que la semántica depende de la pragmática. Aunque tanto James como Peirce aceptaron la importancia de la coherencia de los conceptos en un contexto, ninguno de ellos era un inferencialista en este sentido. A lo más, por adscribirles una doctrina semejante a las contemporáneas, ambos aceptaron una combinación de coherencia y referencia análoga a lo que Susan Haack llama "foundherentism", pues a ambos filósofos les importa una referencia a un control público, objetivo y recalcitrante.

5 James, William, Pragmatism: A New Name for some Old Ways of Thinking, Cambridge: Harvard University Press, 1975, p. 34.

6 Ibid. 


\section{§ 5. La máxima pragmática}

Cuando Peirce habla de pragmatismo como un principio lógico fundamental, este principio lógico se formula como una máxima que ayuda a obtener claridad acerca de proposiciones y conceptos. Peirce tiene un sistema pragmatista de filosofía que se sigue de la aceptación de dicha máxima. Clarificar una proposición es decir qué consecuencias prácticas se siguen de su aceptación, pero Peirce quiso formular este principio de manera que pudiese ser probado lógicamente.

La formulación más temprana y familiar a todos nosotros de la máxima se encuentra en How to Make our Ideas Clear? (1878) y es presentada de este modo: "Consider what effects, which might conceivably have practical bearings, we conceive the object of our conception to have. Then, our conception of those effects is the whole of our conception of the object"'.

Una idea que aparece continuamente en la formulación de la máxima es relativa a los hábitos: hábitos de acción y expectación, y una teoría no humeana de leyes y hábitos. La precisión de la formulación de la máxima conlleva una precisión en la formulación del concepto de hábito.

Hábitos de acción asociados con un concepto prueban el valor pragmático de un concepto. A medida que Peirce desarrolló su filosofía también desarrolló la máxima haciéndola más explícita desde el punto de vista teleológico. Veamos esta formulación más tardía: "The entire intellectual purport of any symbol consists in the total of all general modes of rational conduct which, conditionally upon all the possible different circumstances and desires, would ensue upon the acceptance of the symbol". ${ }^{8}$

Tempranamente Peirce defendió que las creencias son hábitos de acción. Lo que Peirce conserve de esta estrategia es que las creencias son hábitos de acción desde la aceptación de que las proposiciones conllevan hábitos de acción. Un modo en el que podemos entender estos desarrollos en el pensamiento

7 Peirce, C.S., The Essential Peirce, 2 v., Bloomington: Indiana University Press, 1992-1999, I, p. 132.

8 Peirce, C.S., The Essential Peirce, II, p. 346. 
de Peirce se puede notar al observar las distintas versiones de la máxima. Si aceptamos una proposición, por ejemplo, "mi maleta es pesada", podemos clarificar nuestro entendimiento de la proposición generando imperativos del tipo: "no la levantes con prisa" o "usa dos manos antes de tratar de cargarla".

La verdad de una proposición en ese contexto lleva a un imperativo del tipo mencionado. Podemos clarificar un concepto identificando imperativos relevantes. La máxima puede ser utilizada en muchos modos; uno de ellos es tratar de reunir toda la información necesaria, información reflexiva, de modo que podamos usar la clarificación para formular experimentos y evaluaciones que se siguen de la aceptación y entendimiento de una proposición.

No hay contextos en los que podamos derivar consecuencias para conceptos vacíos, y la máxima deriva consecuencias, por tanto, de contextos actuales o incluso posibles. Así pues, usamos la máxima para clarificar proposiciones con un rango limitado de contextos: esta es la mejor manera de interpretar la idea controversial de que las creencias son hábitos de acción.

La máxima identifica cogniciones canónicas: toda y solo la información que es importante sobre una creencia para un propósito en cuestión. Si la máxima funciona para las creencias, también ha de ser derivable para otros elementos de nuestro ajuar cognitivo.

Peirce, sin embargo, tuvo serias dudas sobre considerar a la "creencia" como una cognición fundamental. Su escepticismo se concentró en los años 1900 y 1903, en donde empezó a substituir la idea de "creencia" como la de "juicio". Después, consideró que era mejor hacerlo como la expresión de un juicio, esto es, la "aserción", aunque finalmente cree que la mejor manera de formular la cognición fundamental era la idea de "manifestación del entendimiento".

A la base de estas preocupaciones se encuentra un antipsicologismo creciente. Peirce rehuyó la idea de que las creencias son estados psicológicos y rechazó, por tanto, la idea de una fundamentación psicologista para la cognición. En este sentido el paso de la preferencia en las formulaciones de la máxima por el concepto de "juicio" está basado en la preferencia por la facilidad de formular lógicamente los juicios. La estructura de un juicio es una forma lógica. Sin embargo, los juicios suponen un conocimiento contextual previo que implica 
compromisos no estrictamente lógicos. Por esta razón, Peirce se interesó más y más en mostrar que no se trataba de cualquier idea del juicio, sino la exteriorización de un juicio, es decir, la aserción: esta última puede formularse atendiendo a demandas y críticas por equivocar un juicio.

Peirce se preocupaba por garantizar procesos públicos y transparentes en lo que consideremos como las cogniciones fundamentales; $y$ estas cogniciones fundamentales son los elementos lógicos que pueden ser considerados libres de la ambigüedad psicologística. Ya que la máxima nos ayuda a captar el significado de una aserción, o los elementos lógicos y públicos de un juicio, la máxima también nos ayuda a revelar lo que es importante con fuerza asertórica al creer una proposición. Finalmente, esto le llevó a pensar que los objetos abstractos del pensamiento que conllevan los elementos puramente lógicos se pueden formular como proposiciones. Un ejemplo de este tipo de objetos son las estructuras matemáticas, pues las notaciones que realizamos cuando razonamos matemáticamente producen diagramas que representan e instancian dichas estructuras. Los diagramas, por tanto, son una manifestación de dichos elementos lógicos ya que a través de ellos podemos formular los experimentos necesarios para derivar el entendimiento de los aspectos lógicos implicados en una proposición.

Esta narrativa de los procesos en los que Peirce entendió a la máxima como operante nos proporciona elementos de claridad en donde hay necesidad de revelar elementos lógicos: una "manifestación del entendimiento". La idea temprana de "clarificar nuestras ideas" llega a su culminación por medio de un aprehendimiento reflejo de los conceptos clarificados por la máxima-entendida como principio lógico que revela los elementos lógicos entendidos como hábitos de expectación y acción a través del juicio entendido como un diagrama público y experimental.

\section{$\S 6$. Claridad}

El pragmatismo fue introducido por primera vez en un opúsculo sobre cómo esclarecer ideas; así pues, la primera consecuencia práctica de la máxima misma es la propiedad semántica de la claridad. F. Thomas Burke $(2013,55)$ 
ofrece una lista de los grados de claridad que la máxima permite aplicar'. Me permito reproducirlos a fin de encontrar la vinculación con las perspectivas mencionadas en las que la máxima se prueba a sí misma como un principio lógico:

Claridad, : algo que nos es familiar $^{10}$.

Claridad $_{2}$ : un concepto que nos es precisamente definido en términos abstractos, de los cuales todos son claros en el sentido anterior".

Claridad $_{3}$ : definido operacionalmente, en términos del entendimiento en el que el "razonamiento fructífero" es aplicable "en la resolución de problemas prácticos difíciles"'2.

Claridad $_{4}$ : razonable completa o globalmente, en términos concretos, es decir, incorporado consistentemente con el conjunto de creencias de manera que se vuelve inferencialmente identificable desde las ideas y opiniones que son claras en el sentido 3 .

De estas ideas se sigue que el pragmatismo tiene ambos aspectos, tanto el operacionalista como el inferencialista, derivados de los grados de claridad lógica ofrecidos por el uso de la máxima. Finalmente, hacia 1902 Peirce $^{13}$ ofrece, con su idea del "crecimiento de la racionalidad concreta", una síntesis de los aspectos inferencialista y operacionalista del pragmatismo:

- Concreción: definir nuestros términos de manera operacional para finalmente formular hipótesis experimentales comprobables acordes a las especificaciones operacionales.

- Razonabilidad: se obtiene mediante los procesos autocontrolados de inferencia por los cuales derivamos las especificaciones operacionales, estos procesos son abductivos, deductivos o inductivos.

9 Cf. Burke, Thomas, op. cit., p. 55.

10 Cf. Peirce, C.S., The Essential Peirce, I, pp. 124-125.

II Cf. ibid., pp. I27-I32.

12 Peirce, C.S., The Collected Papers, 6 v., Cambridge: Harvard University Press, III, p. 457.

I3 Cf. Peirce, C.S., The Collected Papers, II, p. 34. 
En conclusión, podemos notar que la diferencia fundamental entre el pragmaticismo peirceano y otras expresiones del pragmatismo tiene como criterio fundamental el uso de la máxima pragmatica como un principio lógico del cual se puede seguir un sistema pragmatista de filosofía. La máxima pragmática, sin embargo, no es ajena al trabajo de otros pragmatistas como James y Dewey, aunque esto no sea evidente. Contar con un principio como la máxima ayuda a reconsiderar la importancia de encontrar un balance adecuado entre el operacionalismo y el inferencialismo que caracterizan metodológicamente el pragmatismo amplio. Tanto Peirce como James tuvieron un énfasis particular propio en cada uno de sus enfoques: Peirce fue decisivamente más operacionalista mientras que James enfatizó las consecuencias inferenciales que se siguen de una teoría inferencialista y, así, enfatizó en una teoría pragmatista de la verdad. Con todo, el énfasis que los pragmatistas clásicos dieron a sus respectivas versiones del pragmatismo emergían naturalmente de una consideración primaria de la máxima, esta lección ayudaría a disipar la ambigüedad presente en versiones contemporáneas del pragmatismo y, por tanto, supone una contribución a un giro pragmatista de la filosofía sustentado más consistentemente. 